\title{
IGF-1 and VEGF can be used as prognostic indicators for patients with uterine fibroids treated with uterine artery embolization
}

\author{
YONGXU MU ${ }^{1,2^{*}}, J_{U N F E N G ~ H E}{ }^{* *}$, RUIQIANG YAN ${ }^{2}$, XIAOYAN HU ${ }^{2}$, HAIYAN LIU $^{2}$ and ZHIMING HAO ${ }^{1}$ \\ ${ }^{1}$ Department of Rheumatism, The First Affiliated Hospital of Xi'an Jiaotong University, Xi'an, Shaanxi 710049; \\ ${ }^{2}$ Department of Intervention, The First Affiliated Hospital of Baotou Medical College, \\ Baotou, Inner Mongolia Autonomous Region 014010, P.R. China
}

Received September 26, 2014; Accepted August 7, 2015

DOI: $10.3892 /$ etm.2015.2924

\begin{abstract}
The aim of the present study was to investigate the association between serum vascular endothelial growth factor (VEGF) and insulin-like growth factor 1 (IGF-1) levels and the prognosis of patients with uterine fibroids following uterine artery embolization (UAE) treatment. A total of 70 patients with uterine fibroids and 20 healthy controls were enrolled in this study between 2012 and 2014. The serum levels of IGF-1 and VEGF were measured using ELISA. Multiple-factor analysis was performed to assess the association between serum levels of IGF-1/VEGF and certain clinical characteristics, including size, location, number of uterine fibroids and adenomyosis. Progression-free survival curves were analyzed using the Kaplan-Meier method. The serum levels of IGF-1 and VEGF in patients with uterine fibroids prior to UAE treatment were significantly higher than those in controls $(\mathrm{P}<0.05)$. At 1 week after UAE treatment, the serum levels of IGF-1 and VEGF were significantly lower compared with those prior to UAE treatment. The serum levels of IGF-1 and VEGF at 1 or 3 months after UAE treatment were significantly higher than those at 1 week after UAE treatment. The serum levels of IGF-1 and VEGF were significantly correlated with the clinical characteristics of uterine fibroids $(\mathrm{P}<0.05)$. Lower levels of IGF-1 and VEGF in the serum following UAE treatment were associated with an enhanced progression-free survival of patients. In conclusion, the levels of IGF-1 and VEGF in the serum following UAE treatment can be used as indicators of prognosis in patients with uterine fibroids.
\end{abstract}

Correspondence to: Dr Zhiming Hao, Department of Rheumatism, The First Affiliated Hospital of Xi'an Jiaotong University, 277 Yanta West Road, Xi'an, Shaanxi 710049, P.R. China

E-mail: mxx333@126.com

*Contributed equally

Key words: uterine fibroid, uterine artery embolization, insulin-like growth factor 1, vascular endothelial growth factor

\section{Introduction}

Uterine fibroids, which have an incidence rate of $20-25 \%$, are the most common benign tumors in females (1). The clinical manifestations of uterine fibroids mainly include menorrhagia, menostaxis, dysmenorrhea and anemia. Clinically, uterine fibroids are mainly treated with surgical therapy (hysterectomy and myomectomy) and hormone therapy $(2,3)$; however, the recurrence rate of uterine fibroids subsequent to these treatments is relatively high, and the trauma caused by these treatments can be extensive. Uterine artery embolization (UAE) is a minimally invasive procedure that offers an alternative to the traditional surgical removal of the uterine fibroids (4). During the procedure, the blood supply of the fibroids is blocked, which leads to the shrinkage of the fibroids. UAE has been widely used in recent years for the treatment of uterine fibroids.

Insulin-like growth factor 1 (IGF-1) and vascular endothelial growth factor (VEGF) play important roles in the growth of uterine fibroids (5). IGF-1, a tumor autocrine growth factor, is highly expressed in numerous types of tumor and has a key role in stimulating cell growth and proliferation (6-8). VEGF is an important growth factor that stimulates the proliferation of vascular endothelial cells. It has been reported that VEGF promotes cell migration and proliferation by binding to the associated receptors (9), thereby affecting angiogenesis (7). Although uterine fibroids are benign tumors, angiogenesis is also critical for its occurrence and development. UAE treatment can induce ischemia and hypoxia of uterine fibroids by obstructing their blood supply (10). It has been found that hypoxia-inducible factor-1 $\alpha$, an important factor regulating oxygen balance, can induce VEGF gene transcription and increase the stability of VEGF mRNA (11), thus elevating the expression level of VEGF and stimulating uterine fibroid angiogenesis.

It has yet to be elucidated whether IGF-1 and VEGF could be used as prognostic factors for patients with uterine fibroids following UAE treatment. In the present study, the serum levels of IGF-1 and VEGF prior to and following UAE treatment were measured, and the effects of IGF-1 and VEGF on progression-free survival were analyzed, in order to provide experimental evidence to enable the evaluation of the prognosis of uterine fibroid patients following UAE treatment. 


\section{Patients and methods}

Clinical data. A total of 70 patients with uterine fibroids, who were hospitalized at the Department of Intervention, the First Affiliated Hospital of Baotou Medical College (Baotou, China) between January 2012 and December 2014, were included in the UAE group and received UAE treatment. The inclusion criteria were as follows: i) Uterine fibroids were diagnosed through clinical gynecological, type-B ultrasound and other auxiliary examinations; ii) patients exhibited a normal coagulation function; iii) patients were free from underlying diseases, comorbidities or complications; and iv) the age of the patients was $\leq 60$ years. The exclusion criteria were as follows: i) Patients not meeting the inclusion criteria; ii) presence of lesions in other parts of the body or intraoperative complications; iii) patients with cervical and broad ligament fibroids; and iv) patients with abnormal coagulation function. The age of the 70 patients in the UAE group ranged from 27 to 60 years, with an average age of $39.6 \pm 7.69$ years. Among the patients in this group, 25 were single and 45 were married. A total of 47 patients exhibited menorrhagia and menostaxis, and 22 patients suffered from pain in the lower abdomen and waist. Compression symptoms, such as urinary frequency, urinary urgency and constipation, were found in 54 cases, and anemia was found in 39 cases. A total of 20 healthy individuals booked in for random health checks were additionally included in the study as a control group. The Karnofsky Performance Scale score of patients was evaluated following admission to the hospital (12).

The patients were followed-up at 1 week and 3, 6 and 18 months after UAE treatment. The size of the fibroids and uterus were evaluated by ultrasound, computed tomography or magnetic resonance imaging examination. Progression-free survival referred to the period between the diagnosis of the tumor and the end of the follow-up in patients without recurrence and regeneration of fibroids. A total of 21 patients did not complete the study, as a result of being lost to follow-up at the time of last contact or prior to the study cut-off or due to succumbing to an unrelated cause. These cases were censored.

Prior written and informed consent was obtained from every patient, and the study was approved by the Ethics Review Board of the First Affiliated Hospital of Baotou Medical College.

Sample preparation. Peripheral blood $(4 \mathrm{ml})$ was collected from cubital veins prior to UAE treatment and at 1 week and 3 and 6 months after UAE treatment. Serum was isolated by centrifugation at $1,411 \times \mathrm{g}$ for $15 \mathrm{~min}$ and then stored at $-40^{\circ} \mathrm{C}$ until further analysis.

UAE treatment. UAE treatment was performed by two physicians as previously described (13). Briefly, catheterization was performed through the right femoral artery under local anesthesia (5 ml lidocaine hydrochloride; Tianjin Jin Yao Amino Acid Co., Ltd., Tianjin, China). Then, contralateral iliac artery angiography and uterine artery angiography was performed (Artis dTC Angiography; Siemens AG, Munich, Germany). Following the elimination of key branches of the uterine artery, the emulsified mixture of lipiodol $(5 \mathrm{ml}$; Guerbet, Villepinte, France) and pingyangmycin (4 mg; Shanghai Yansheng Shiye
Table I. Clinical data of patients with uterine fibroids.

\begin{tabular}{lcr}
\hline Clinical features & Average (range) & Cases, $\mathrm{n}$ \\
\hline Age, years & $39.6 \pm 7.69(25-60)$ & 7 \\
$\geq 50$ & & 63 \\
$<50$ & & 8 \\
Adenomyosis of uterus & & 62 \\
Yes & & \\
No & & \\
Location of fibroids & 17 & \\
Intramural & 11 & \\
Submucosal & & \\
Subserosal & & \\
Size of fibroids, cm & $3.97 \pm 1.48(1.5-6.5)$ & \\
$\geq 1.5$ & & 31 \\
$<6.5$ & & 39 \\
Number of fibroids & & \\
Solitary & & \\
Multiple & & \\
\hline
\end{tabular}

Co., Ltd. Shanghai, China) was injected into the uterine artery. After the majority of the uterus was dyed and the blood flow reduced, gelatin sponge $(10 \times 2 \times 2 \mathrm{~mm})$ was used to embolize the uterine artery. The embolization was verified by angiography. The embolization of the ipsilateral uterine artery was performed accordingly.

ELISA. ELISA assay was performed using IGF-1 (YM-S2154) and VEGF165 (YM-10632) ELISA kits (Shanghai YuanMu Biological Technology Co., Ltd., Shanghai, China) according to the manufacturer's instructions. Briefly, serum was added to the pre-coated microplate and incubated at $4{ }^{\circ} \mathrm{C}$ overnight. The plate was then washed 5 times with phosphate-buffered saline and the detection antibody was added to the wells. Following incubation at room temperature for $1 \mathrm{~h}$, the microplate was washed again, and horseradish peroxidase-conjugated antibody was added and incubated at room temperature for $30 \mathrm{~min}$. All antibodies were from the IGF-1 and VEGF165 ELISA kits. After $30 \mathrm{~min}$, the plate was re-washed 5 times, $o$-phenylenediamine (OPD) substrate solution was added and the plate was incubated at room temperature for a further 15 min. OPD was part of the IGF-1 and VEGF165 ELISA kits. Finally, stop solution was added to terminate color development and the plate was read at $450 \mathrm{~nm}$ on a microplate reader (SpectraMax M2; Molecular Devices LLC, Sunnyvale, CA, USA). The standard curve was generated using 2-fold serial dilutions of the standard samples. Levels of IGF-1 and VEGF were calculated according to the standard curve.

Statistical analysis. Results are expressed as the mean \pm standard deviation. All statistical analyses were performed with SPSS version 17.0 for Windows (SPSS, Inc., Chicago, IL, USA). Comparisons between groups and analyses of paired data were conducted using the Student's t-test. Multiple-factor analysis was performed to analyze the association between levels of 
Table II. Levels of IGF-1 and VEGF in patients with uterine fibroids before and after UAE treatment.

\begin{tabular}{|c|c|c|c|c|}
\hline Groups & IGF-1 (pg/ml) & P-value & VEGF (pg/ml) & P-value \\
\hline Control & $122.1 \pm 46.4$ & & $77.9 \pm 34.8$ & \\
\hline \multicolumn{5}{|l|}{ UAE } \\
\hline Before UAE & $134.5 \pm 45.1^{\mathrm{a}}$ & 0.025 & $144.0 \pm 56.6^{\mathrm{a}}$ & $<0.001$ \\
\hline 1 week after UAE & $70.2 \pm 20.4^{\mathrm{b}}$ & 0.001 & $86.2 \pm 33.3^{\mathrm{b}}$ & 0.001 \\
\hline 1 month after UAE & $118.3 \pm 48.8^{c}$ & $<0.001$ & $109.5 \pm 42.7^{\mathrm{c}}$ & $<0.001$ \\
\hline 3 months after UAE & $131.3 \pm 43.1^{\mathrm{c}}$ & $<0.001$ & $136.7 \pm 52.6^{c}$ & 0.015 \\
\hline
\end{tabular}

Compared with control group, ${ }^{\mathrm{P}} \mathrm{P}<0.05$. Compared with UAE group before UAE treatment, ${ }^{\mathrm{b}} \mathrm{P}<0.05$. Compared with UAE group at 1 week after UAE treatment, ${ }^{\mathrm{P}} \mathrm{P}<0.05$. IGF-1, insulin-like growth factor 1; VEGF, vascular endothelial growth factor; UAE, uterine artery embolization.

Table III. Association between the serum levels of IGF-1 or VEGF and clinical characteristics of uterine fibroids.

\begin{tabular}{|c|c|c|c|c|c|}
\hline $\begin{array}{l}\text { Clinical } \\
\text { characteristics }\end{array}$ & Cases & $\begin{array}{l}\text { IGF-1 }(\mathrm{pg} / \mathrm{ml}) \\
\text { at } 1 \text { week after } \\
\text { UAE treatment }\end{array}$ & P-value & $\begin{array}{c}\text { VEGF }(\mathrm{pg} / \mathrm{ml}) \text { at } \\
1 \text { week after UAE } \\
\text { treatment }\end{array}$ & P-value \\
\hline Adenomyosis of uterus & & & 0.002 & & 0.048 \\
\hline Yes & 8 & $96.2 \pm 8.51$ & & $77.6 \pm 13.6$ & \\
\hline No & 62 & $66.9 \pm 23.7$ & & $87.4 \pm 35.0$ & \\
\hline Location of fibroids & & & 0.041 & & 0.032 \\
\hline Intramural & 42 & $60.5 \pm 21.4$ & & $71.5 \pm 21.5$ & \\
\hline Submucosal & 17 & $81.3 \pm 25.5$ & & $104.7 \pm 33.8$ & \\
\hline Subserosal & 11 & $90.3 \pm 10.3$ & & $114.1 \pm 39.9$ & \\
\hline Number of fibroids & & & 0.043 & & 0.093 \\
\hline Solitary & 31 & $84.3 \pm 17.9$ & & $86.1 \pm 27.6$ & \\
\hline Multiple & 39 & $60.2 \pm 22.7$ & & $86.4 \pm 37.6$ & \\
\hline Size of fibroids, $\mathrm{cm}$ & & & 0.006 & & 0.005 \\
\hline$\geq 1.5$ & 58 & $65.6 \pm 23.7$ & & $79.8 \pm 25.2$ & \\
\hline$<6.5$ & 12 & $92.8 \pm 11.6$ & & $117.3 \pm 49.0$ & \\
\hline Age, years & & & 0.054 & & 0.201 \\
\hline$\geq 50$ & 7 & $88.1 \pm 141$ & & $95.3 \pm 16.2$ & \\
\hline$<50$ & 63 & $68.3 \pm 24.5$ & & $85.2 \pm 33.3$ & \\
\hline
\end{tabular}

IGF-1, insulin-like growth factor 1; VEGF, vascular endothelial growth factor; UAE, uterine artery embolization.

IGF-1 or VEGF and certain clinical characteristics, including adenomyosis and the age of the patients, as well as fibroid size, location and number. The Kaplan-Meier method was used to assess the progression-free survival of the patients. The differences in survival time were analyzed using a Log-Rank test. $\mathrm{P}<0.05$ was considered to indicate a statistically significantly difference.

\section{Results}

General data of patients with uterine fibroids. The general data of the patients with uterine fibroids are shown in Table I. Among the 70 patients, 53 were unable or not willing to undergo surgery and 17 were patients who had recurrent uterine fibroids following surgical removal. There were 31 patients with a solitary fibroid and 39 with multiple fibroids. The fibroids were located as follows: 42, intramural; 17, submucosal; and 11, subserosal. Eight patients with uterine fibroids exhibited concurrent adenomyosis. In all 70 patients, the Karnofsky score was $\geq 90$.

Serum levels of IGF-1 and VEGF prior to and following UAE treatment. To determine the levels of IGF-1 and VEGF in the serum, ELISA was performed prior to and following the UAE treatment. As shown in Table II, the serum levels of IGF-1 and VEGF in the UAE group prior to treatment were $134.5 \pm 45.1$ and $144.0 \pm 56.6 \mathrm{pg} / \mathrm{ml}$, respectively; these levels were significantly higher than those in the control group $(122.1 \pm 46.4 \mathrm{pg} / \mathrm{ml}$ for IGF-1 and 77.9 $\pm 34.8 \mathrm{pg} / \mathrm{ml}$ for VEGF) $(\mathrm{P}<0.05)$. At 1 week after UAE treatment, the serum levels of IGF-1 and VEGF in the patients of the UAE group were $70.2 \pm 20.4$ and $86.2 \pm 33.3 \mathrm{pg} / \mathrm{ml}$, respectively. Compared with the levels 

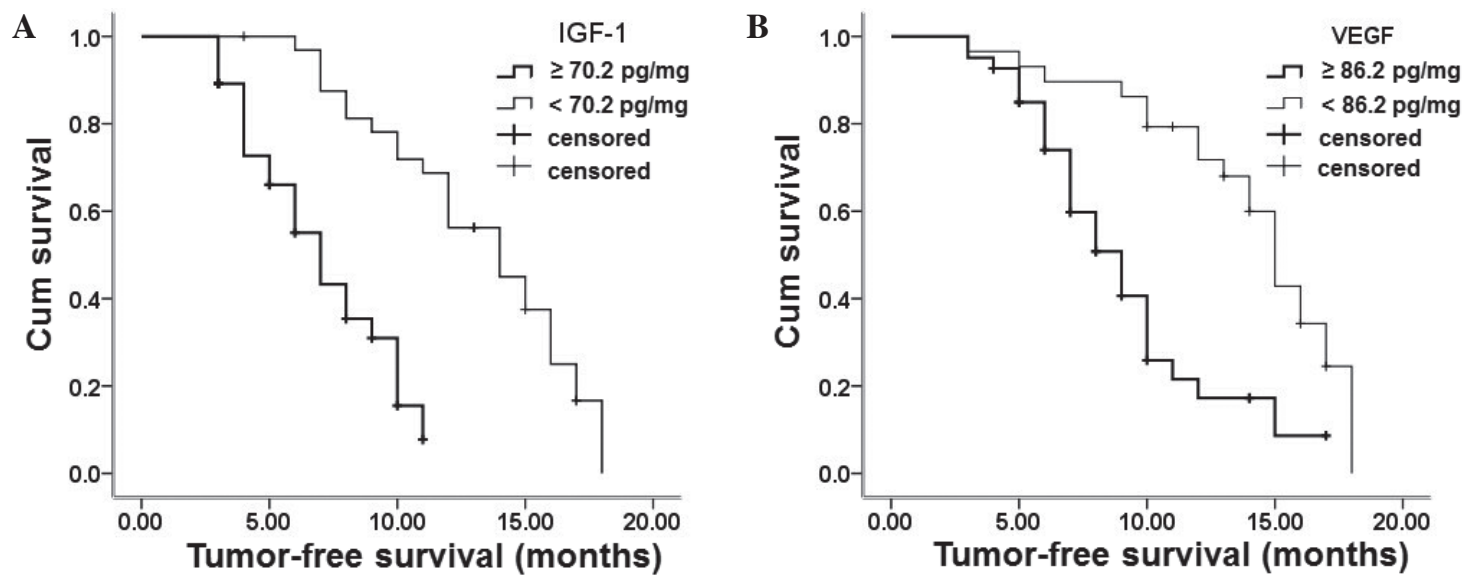

Figure 1. Survival analysis of uterine fibroid patients with different levels of (A) IGF-1 and (B) VEGF in the serum. The median levels of IGF-1 and VEGF were 70.2 and $86.2 \mathrm{pg} / \mathrm{ml}$, respectively. Patients were followed-up for 18 months. During the follow-up, 21 cases were censored. A Kaplan-Meier survival curve was generated, the differences in survival time were analyzed using the Log-Rank test. IGF-1, insulin-like growth factor 1; VEGF, vascular endothelial growth factor.

prior to UAE treatment, the serum levels of IGF-1 and VEGF at 1 week after UAE treatment were significantly lower $(\mathrm{P}<0.05)$. At 1 month after UAE treatment, the serum levels of IGF-1 $(118.3 \pm 48.8 \mathrm{pg} / \mathrm{ml})$ and VEGF $(109.5 \pm 42.7 \mathrm{pg} / \mathrm{ml})$ were significantly higher than those at 1 week after UAE treatment $(\mathrm{P}<0.05)$. Similarly, the serum levels of IGF-1 $(131.3 \pm 43.1 \mathrm{pg} / \mathrm{ml})$ and VEGF $(136.7 \pm 52.6 \mathrm{pg} / \mathrm{ml})$ at 3 months after UAE treatment were significantly higher than those at 1 week after UAE treatment $(\mathrm{P}<0.05)$.

Association between the serum levels of IGF-1 and VEGF and the clinical characteristics of patients with uterine fibroids. To investigate the association between the serum levels of IGF-1 and VEGF at 1 week after UAE treatment and the clinical characteristics of the patients with uterine fibroids, multiple-factor analysis was performed. The analyzed clinical characteristics included the age of the patient, adenomyosis and the size, location and number of fibroids. As shown in Table III, the serum IGF-1 level at 1 week after UAE treatment significantly differed according to the clinical characteristics of adenomyosis and the size, location and number of fibroids $(\mathrm{P}<0.05)$. No significant difference was found in the serum IGF-1 level between different age groups $(\mathrm{P}>0.05)$. The serum VEGF level at 1 week after UAE treatment significantly differed according to the clinical characteristics of adenomyosis and the size and location of the fibroids $(\mathrm{P}<0.05)$. No significant differences in serum VEGF levels were found between patients with different numbers of fibroids or different ages $(\mathrm{P}>0.05)$. These results suggest that IGF-1 and VEGF may be used for predicting the prognosis of patients with uterine fibroids.

Effect of serum IGF-1 and VEGF levels on progression-free survival of patients with uterine fibroids. To investigate the effect of IGF-1 and VEGF serum levels on the prognosis of patients with uterine fibroids, the Kaplan-Meier method was performed to analyze the progression-free survival of the patients. The patients were followed-up for 18 months. Progression-free survival curves were constructed based on the serum levels of IGF-1 and VEGF at 1 week after UAE treatment (70.2 and $86.2 \mathrm{pg} / \mathrm{ml}$, respectively) and are shown in Fig. 1. A total of 37 patients had a serum IGF-1 level $\geq 70.2 \mathrm{pg} / \mathrm{ml}$ and 33 patients had a serum IGF-1 level $<70.2 \mathrm{pg} / \mathrm{ml}$. The progression-free survival of the patients with a serum IGF-1 level $\geq 70.2 \mathrm{pg} / \mathrm{ml}$ was 1.5 months, which was significantly shorter than that of patients with a serum IGF-1 level $<70.2 \mathrm{pg} / \mathrm{ml}$ (3 months). The number of patients with serum VEGF levels $\geq 86.2$ and $<86.2 \mathrm{pg} / \mathrm{ml}$ was 29 and 41 , respectively. The progression-free survival of patients with a serum VEGF level $\geq 86.2 \mathrm{pg} / \mathrm{ml}$ was 2 months, which was significantly shorter than that of patients with a serum IGF-1 level $<86.2 \mathrm{pg} / \mathrm{ml}$ (4 months). These results indicate that patients with higher levels of IGF-1 and VEGF have a poorer prognosis than those with lower IGF-1 and VEGF levels.

\section{Discussion}

The main blood supply of uterine fibroids is from the uterine artery and its branches. UAE treatment can induce ischemia and atrophy of the uterine fibroids (14) and is less invasive than the traditional surgical approach. Furthermore, UAE treatment can successfully preserve the uterus and maintain its normal physiological functions $(15,16)$. IGF-1 and VEGF are important factors involved in the growth of uterine fibroids (5).

In the present study, the serum levels of IGF-1 and VEGF in the UAE group prior to UAE treatment were significantly higher than those in the control group. At 1 week after UAE treatment, the serum IGF-1 and VEGF levels in the UAE group were significantly decreased compared with those prior to UAE treatment; however, at 1 and 3 months after UAE treatment, the serum IGF-1 and VEGF levels were significantly increased compared with those at 1 week after UAE treatment. These results were consistent with the findings of a previous study by Ji et al (17). The decrease in the serum level of IGF-1 at 1 week after UAE treatment may have been due to the temporary inhibition of IGF-1 expression. At 1 and 3 months after UAE treatment, the serum level of IGF-1 was increased. These results suggest that IGF-1 plays an important role in the growth of fibroids. The decrease in the serum level of VEGF following UAE treatment may have been due to the ischemia and hypoxia after the embolization. These findings therefore 
indicate that IGF-I and VEGF can be used as indicators of prognosis following UAE treatment.

This study further analyzed the association between serum levels of IGF-1 and VEGF and the clinical factors associated with the therapeutic effect of uterine fibroid treatment, including the size, location and number of uterine fibroids, as well as the age of the patient and the presence of adenomyosis. The serum levels of IGF-1 and VEGF were significantly different among patients with different sizes and locations of uterine fibroids, as well as between patients with and without adenomyosis. These data suggest that IGF-1 and VEGF have an important effect on the prognosis associated with uterine fibroids.

To further verify the association between serum IGF-1 and VEGF levels and the prognosis of patients with uterine fibroids, the progression-free survival of the patients was analyzed using the Kaplan-Meier method. The median levels of IGF-1 and VEGF were calculated according to the serum levels of IGF-1 and VEGF at 1 week after UAE treatment. Patients were divided into two groups according to whether their serum IGF-1 (or VEGF) level was higher or lower than the median level. The results showed that, at the end of follow-up, the progression-free survival of patients with serum levels of IGF-1 and VEGF lower than the median level was significantly enhanced. These data suggest that lower levels of IGF-1 and VEGF in the serum following UAE treatment indicate a good prognosis.

In conclusion, the serum levels of IGF-1 and VEGF exhibited significant changes prior to and following UAE treatment. Furthermore, the serum levels of IGF-1 and VEGF at 1 week after UAE treatment were significantly different among patients with different uterine fibroid-related clinical characteristics. Additionally, patients with lower levels of IGF-1 and VEGF in the serum following UAE treatment had significantly longer progression-free survival. Based on these results, IGF-1 and VEGF may be used as prognostic factors for evaluating the prognosis of patients with uterine fibroids following UAE treatment.

\section{Acknowledgements}

This study was supported by a grant from Baotou Science and Technology Bureau Intellectual Property (no. BK2008123).

\section{References}

1. Okogbo FO, Ezechi OC, Loto OM and Ezeobi PM: Uterine Leiomyomata in South Western Nigeria: A clinical study of presentations and management outcome. Afr Health Sci 11: 271-278, 2011.
2. Zhang Y, Sun L, Guo Y, Cheng J, Wang Y, Fan S and Duan H: The impact of preoperative gonadotropin-releasing hormone agonist treatment on women with uterine fibroids: A meta-analysis. Obstet Gynecol Surv 69: 100-108, 2014.

3. Wang A and Yin S: Controversy and challenge in the treatment methods of uterine fibroids. Zhong Guo Quan Ke Yi Xue 12: 14-15, 2009 (In Chinese).

4. Singh C, Gupta M, Tripathi R and Tyagi S: Successful use of transcatheter embolisation in an emergent life-threatening situation of bleeding from uterine arteriovenous malformation. BMJ Case Rep 2013, 2013.

5. Mathonnet M, Descottes B, Valleix D, Labrousse F, Truffinet V and Denizot Y: Quantitative analysis using ELISA of vascular endothelial growth factor and basic fibroblast growth factor in human colorectal cancer, liver metastasis of colorectal cancer and hepatocellular carcinoma. World J Gastroenterol 12: 3782-3783, 2006.

6. Fan ZR, Yang DH, Zuo HR, Xu Z and Qiu QL: Expressions of IGF-I, IGF-II and their receptor mRNAs in hepatocellular carcinomas and adjacent non-tumor tissues. Ai Zheng 19: 150-152,155, 2000 (In Chinese).

7. Pourgholami MH and Morris DL: Inhibitors of vascular endothelial growth factor in cancer. Cardiovasc Hematol Agents Med Chem 6: 343-347, 2008

8. Dai B, Wang DS, Zhang Y, Zhou L, Liu J and Sun W: The role of insulin-like growth factors in tumor. Zhong Hua Lin Chuang Yi Shi Za Zhi (Dian Zi Ban) 7: 7099-7102, 2013 (In Chinese)

9. Ding ZX, Zhang LZ and Ling L: Levels of matrix metalloproteinase and vascular endothelial growth factor in patients with primary liver cancer. Gan Zang 10: 214-215, 2005 (In Chinese).

10. Liao D, Fan Z and Huang R: Clinical Report of treating uterine fibroids and uterine adenomyosis with uterine artery embolization. Wei Chuang Yi Xue 7: 256-258, 2012 (In Chinese).

11. Gu J, Guan Q, Ji W and Ren W: The influence of serum HIF-1a and VEGF content on the prognosis in patients with hepatocellular carcinoma after TACE. Jie Ru Fang She Xue Za Zhi 23: 142-146, 2014 (In Chinese)

12. Marina O, Suh JH, Reddy CA, Barnett GH, Vogelbaum MA, Peereboom DM, Stevens GH, Elinzano H and Chao ST: Treatment outcomes for patients with glioblastoma multiforme and a low Karnofsky Performance Scale score on presentation to a tertiary care institution. Clinical article. J Neurosurg 115: 220-229, 2011.

13. Ahmad A, Qadan L, Hassan N and Najarian K: Uterine artery embolization treatment of uterine fibroids: effect on ovarian function in younger women. J Vasc Interv Radiol 13: 1017-1020, 2002.

14. Pelage JP, Cazejust J, Pluot E, Le Dref O, Laurent A, Spies JB, Chagnon $S$ and Lacombe P: Uterine fibroid vascularization and clinical relevance to uterine fibroid embolization. Radiographics 25 (Suppl 1): S99-S117, 2005.

15. Wang L: Clinical study of treating uterine fibroids with uterine artery embolization. Zhong Guo Yi Yao 2: 238-241, 2012 (In Chinese).

16. Qu W, Guo P and Wu Y: Clinical research of uterine artery embolization for symptomatic hysteromyoma. J Pract Med Tech 18: 573-576, 2011.

17. Ji W, Xin X and Chen B: The influence of insulin-like growth factor 1 and insulin-like growth factor 1 receptor on the growth of uterine leiomyoma. Zhong Guo Zhong Liu Lin Chuang Yu Kang Fu 10: 24-26, 2003 (In Chinese). 